Mir143/145 gene cluster alter homeostatic control of blood pressure, and are miRNAs involved in pathological conditions including atherosclerosis? The answers to these questions promise to provide new insights into our understanding of cardiovascular development and the pathogenesis of vascular proliferative syndromes.

\section{Acknowledgments}

This commentary was supported in part by NIH grants R01 HL094520 and P01 HL075215 and the Commonwealth of Pennsylvania.

Address correspondence to: Michael S. Parmacek, University of Pennsylvania School of Medicine, 9035 Gates Pavilion, 3400 Spruce St., Philadelphia, Pennsylvania 19104, USA. Phone: (215) 662-3140; Fax: (215)349-8017;E-mail: michael.parmacek@ uphs.upenn.edu.

1. Owens, G.K., Kumar, M.S., and Wamhoff, B.R 2004. Molecular regulation of vascular smooth muscle cell differentiation in development and disease. Physiol. Rev. 84:767-801.
2. Schwartz, S.M. 1997. Smooth muscle migration in atherosclerosis and restenosis. J. Clin. Invest. 100:S87-S89.

3. Majesky, M.W. 2009. Developmental biology in the vasculature - review series. Arterioscler. Thromb. Vasc. Biol. 29:622.

4. Parmacek, M.S. 2007. Myocardin-related transcription factors: critical coactivators regulating cardiovascular development and adaptation. Circ. Res. 100:633-644.

5. Sun,Q.,etal.2006. Definingthemammalian CArGome. Genome Res. 16:197-207.

6. Du, K.L., et al. 2003. Myocardin is a critical serum response factor cofactor in the transcriptional program regulating smooth muscle cell differentiation. Mol. Cell. Biol. 23:2425-2437.

7. Yoshida, T., Kawai-Kowase, K., and Owens, G.K. 2004. Forced expression of myocardin is not sufficient for induction of smooth muscle differentiation in multipotential embryonic cells. Arterioscler. Thromb. Vasc. Biol. 24:1596-1601.

8. McDonald, O.G., Wamhoff, B.R., Hoofnagle, M.H., and Owens, G.K. 2006. Control of SRF binding to CArG box chromatin regulates smooth muscle gene expression in vivo. J. Clin. Invest. 116:36-48.

9. Zhou, J., et al. 2009. The SWI/SNF chromatin remodeling complex regulates myocardin-induced smooth muscle-specific gene expression. Arterioscler. Thromb. Vasc. Biol. 29:921-928.

10. Li, S., Wang, D.Z., Wang, Z., Richardson, J.A., and Olson, E.N. 2003. The serum response factor coactivator myocardin is required for vascular smooth muscle development. Proc. Natl. Acad. Sci. U. S. A. 100:9366-9370.
11. Huang, J., et al. 2008. Myocardin regulates expression of contractile genes in smooth muscle cells and is required for closure of the ductus arteriosus in mice. J. Clin. Invest. 118:515-525.

12. Long, X., Creemers, E.E., Wang, D.Z., Olson, E.N., and Miano, J.M. 2007. Myocardin is a bifunctional switch for smooth versus skeletal muscle differentiation. Proc. Natl. Acad. Sci. U. S. A. 104:16570-16575.

13. Boettger, T., et al. 2009. Acquisition of the contractile phenotype by murine arterial smooth muscle cells depends on the Mir143/145 gene cluster. J. Clin. Invest. 119:2634-2647.

14. Cordes, K.R., et al. 2009. miR-145 and miR-143 regulate smooth muscle cell fate and plasticity. Nature. Online publication ahead of print. doi:10.1038/nature08195

15. Bartel, D.P. 2009. MicroRNAs: target recognition and regulatory functions. Cell. 136:215-233.

16. Rajewsky, N. 2006. microRNA target predictions in animals. Nat. Genet. 38(Suppl.):S8-S13.

17. Ivey, K.N., et al. 2008. MicroRNA regulation of cell lineages in mouse and human embryonic stem cells. Cell Stem Cell. 2:219-229.

18. Takahashi, K., et al. 2007. Induction of pluripotent stem cells from adult human fibroblasts by defined factors. Cell. 131:861-872.

19. Schieffer, B., et al. 2000. Expression of angiotensin II and interleukin 6 in human coronary atherosclerotic plaques: potential implications for inflammation and plaque instability. Circulation. 101:1372-1378

20. Pipes, G.C., et al. 2005. Stem cells and their derivatives can bypass the requirement of myocardin for smooth muscle gene expression. Dev. Biol. 288:502-513.

\title{
Defining a role for the homeoprotein Six1 in EMT and mammary tumorigenesis
}

\author{
Derek C. Radisky
}

\author{
Department of Cancer Biology, Mayo Clinic, Jacksonville, Florida, USA.
}

\begin{abstract}
Homeobox (Hox) genes encode transcription factors that act as critical regulators of growth and differentiation during embryogenesis. While many studies have identified increased expression of Hox genes in tumors, much less is known about the mechanistic basis by which Hox genes facilitate tumor development. In this issue of the JCI, McCoy and colleagues show that transgenic mice that express the homeoprotein Six 1 in mammary epithelial cells show increases in stem/progenitor cell populations and subsequent tumor development, while in a separate study Micalizzi and colleagues show that overexpression of $\mathrm{Six} 1$ facilitates breast cancer cell metastasis by inducing epithelial-mesenchymal transition (EMT) (see the related articles beginning on pages 2663 and 2678, respectively). Their findings implicate Six1 as a central mediator of breast cancer development.
\end{abstract}

Breast cancer progression is a highly orchestrated process that is much more than just acquisition of increased cellular proliferative capacity. The first step in the development of breast cancer, proliferation

Conflict of interest: The author has declared that no conflict of interest exists.

Citation for this article: J. Clin. Invest. 119:2528-2531 (2009). doi:10.1172/JCI40555. of epithelial cells into the central lumen, involves the acquisition by the cancer cells of the ability to evade structural and biochemical constraints imposed by cell-cell and cell-extracellular matrix interactions. Progression from early, proliferative disease through ductal carcinoma in situ to invasive breast cancer requires the ability to break down the basement membrane and to interact with the stromal extracellular matrix. The ultimate development of metastatic capability, the most deadly and least treatable stage of breast cancer progression, requires the cancer cells to separate from the parental tumor mass, to intravasate into and subsequently extravasate from the vasculature, and to settle and grow at a distant site. All of these steps, from initial breakdown of tissue structure, through increased invasiveness, and ultimate distribution throughout the body, are characteristic features of epithelial-mesenchymal transition (EMT), a developmental process in which adhesive epithelial cells acquire the motile and migratory characteristics of mesenchymal cells (1). Both developmental and pathologic EMT are associated with dramatic alterations in patterns of gene expression, and recent studies have shown that a number of the transcription factors that regulate developmental EMT, including Snail, Slug, and Twist, are also activated during breast cancer progression (2). 
Normal mammary epithelium

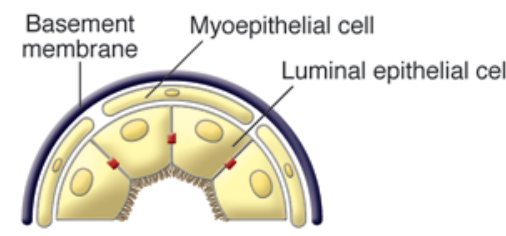

ii Primary mammary epithelial tumor

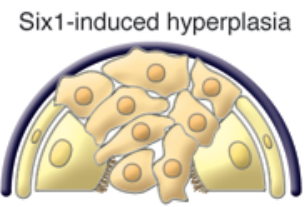

iii EMT

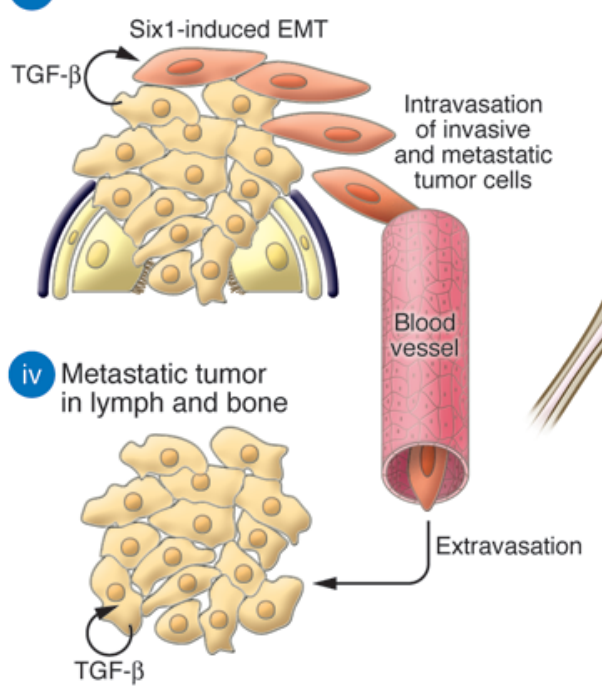

\section{Figure 1}

Mammary tumor growth and metastasis is induced by overexpression of Six1. (i) Normal mammary epithelium consists of a bilayered epithelial structure of myoepithelial and luminal epithelial cells, surrounded by a continuous basement membrane. (ii) In their study in this issue of the $\mathrm{JCl}$, McCoy et al. (15) show that transgenic mice that express Six 1 under control of the mouse mammary tumor virus show induction of mammary ductal hyperplasia and growth of mammary tumors. Micalizzi et al. (16) in their study show that overexpression of Six 1 in cultured human mammary epithelial cells leads to induction of EMT through stimulation of the TGF- $\beta$ receptor and that, following xenograft implantation in immunecompromised mice, Six1-induced EMT facilitates tissue invasion and spread of these cells to the vasculature (iii) and formation of metastases in the lymph and bone (iv).
Determination that transcription factors involved in early stages of development also become reactivated in tumors provides the possibility of entirely novel targets for therapeutic intervention. However, although there has been considerable recent research effort directed toward understanding how EMT is controlled during breast cancer progression, it is clear that our understanding of this process is still incomplete.

\section{EMT as a source of cellular diversity}

Apart from tissue morphogenesis, EMT in development plays an important first step in the generation of diverse cell and tissue types, as cells that have undergone EMT and migrated to different points within the body can redifferentiate into epithelial cells (a process known as mesenchymal-epithelial transition [MET]) or into other cell types (1). Gastrulation, the earliest EMT in development, results in the formation, from the ectoderm, of the mesoderm and the endoderm. The mesoderm contains precursors that give rise to muscle, bone, and connective tissues, while the endoderm ultimately differentiates into organs of the digestive and respiratory tract. EMT that occurs during neural crest delamination produces cells that act as precursors of neurons, melanocytes, bone, and connective tissues. Recent studies have shown that EMT may act in tumor development in a similar fashion, producing cells with stem/progenitor cell characteristics and an increased ability to differentiate into the variety of different cell types that compose the tumor (3-5).

As the initiating cells that give rise to every different cell type in the body, stem cells have been extensively investigated for their role in embryonic development, but studies have shown that cells with stem/ progenitor cell properties may also play critical roles in tumor formation and progression (6). Normal stem cells proliferate very slowly, exist as minority populations in defined niches within tissues, and have distinct responses to cytotoxic agents and other extracellular stimuli $(7,8)$. In normal tissues, stem cells can give rise to other stem cells or to progenitor cells that further differentiate into the different cell types within the tissue. While it has been long suspected that some cancers may be derived from transformed stem cells, or that cancers might acquire the ability of stem cells to undergo limitless proliferation, it was not until 1994 that cells with these properties, designated "tumor-initiating cells" or "cancer stem cells" (CSCs), were first found in human acute myeloid leukemia (9). Since this initial observation, cells with CSC characteristics have been found in solid tumors, including breast, brain, colon, and pancreas (10-14), 
and the evidence that CSCs possess both greatly enhanced tumorigenic capability and increased resistance to many cytotoxic therapies has greatly increased interest in how CSCs are derived and how therapeutic agents more effective for targeting CSCs might be identified (6). Recent investigations using cultured human breast cancer cells $(3,4)$ and transgenic mice $(5)$ have shown that conditions that induce EMT in cancer cells also increase the population of cells with CSC characteristics, providing potential insight into a new mechanism by which EMT may promote tumor progression. Unanswered by these studies, however, was the question of what underlying processes govern the earliest stages of tumor development. A pair of studies in this issue of the JCI, from the research group led by Heide L. Ford, use a transgenic mouse model to show that targeted expression in mammary epithelial cells of Six 1, a protein previously shown to be associated with breast and other cancers, is sufficient to induce EMT, tumor development, and amplification of cells with CSC characteristics $(15,16)$.

\section{Six1 stimulates mammary tumor progression through induction of EMT}

Homeobox (Hox) genes encode an extensive family of conserved transcription factors designated as homeoproteins, members of which have been implicated in nearly every step of embryonic development, controlling proliferation, cell death, and morphogenesis. Deregulation of Hox gene expression has been found in many cancer types, and while a direct causative relationship between increased expression and cancer development has not been identified for most Hox genes, those found to be upregulated in cancer are usually associated with poorly differentiated cell types during development (17). Six1 is a homeoprotein that is essential for the development of a number of organs and is upregulated in proliferating precursor populations relative to differentiated adult tissues (18). Six1 was first identified as upregulated in breast cancer cells in 1998 (18). In the decade since, subsequent studies have shown that it may play a critical role in breast cancer development. Six1 shows increased expression in human breast cancer through gene amplification $(18,19)$, and exogenous overexpression of Six 1 in mammary cells is sufficient to induce malignant transformation and chromosomal instability (20). Mecha- nistic insights come from the observation that overexpression of Six 1 leads to abrogation of cell cycle checkpoints (18). The current studies by McCoy et al. (15) and Micalizzi et al. (16) considerably extend these previous investigations by using mouse models to define how Six 1 induces EMT to stimulate tumor development and metastasis (Figure 1).

McCoy et al. (15) present evidence revealing how Six1 may function to promote tumors at the earliest stages of development through the use of new transgenic mice that inducibly express Six 1 in mouse mammary epithelial cells. Expression of Six1 led to epithelial hyperplasia and alveolar expansion within the first few weeks. Sustained expression of Six 1 led to the formation of aggressive tumors characterized by highly divergent epithelial differentiation that included regions displaying evidence of EMT. Six1 expression was also shown to increase the fraction of epithelial cells expressing mammary stem/progenitor characteristics: isolated primary epithelial cells from Six1-expressing mice showed increased expression of the stem/progenitor cell-associated cell surface markers CD24 and CD29, as well as greatly increased growth as mammospheres, an assay that reflects cell self-renewal. Thus, Six1-induced EMT was associated with increases in the population of stem/progenitor cells and spontaneous tumor growth. Micalizzi et al. (16) used a parallel approach to define how Six1-induced EMT facilitates tumor metastasis using xenograft assays. Overexpression of Six 1 in cultured human mammary cells caused immediate morphological EMT, and Six1-overexpressing cells showed a greatly increased propensity for spontaneous metastasis in orthotopic xenografts, as well as substantially greater metastatic capability following intracardiac injection. Analysis of transcriptional alterations in Six1-overexpressing cells revealed the activation of a TGF- $\beta$ response signature, and inhibition of TGF- $\beta$ signaling in Six1-overexpressing cells greatly reduced their metastatic capability. Consistent with the concept that Six 1 could act to promote metastasis in human tumors, analysis of publicly available datasets showed that increased expression of Six 1 was predictive of decreased time to metastasis, relapse, and survival in breast cancer, and of poor prognosis in a number of other cancers.

\section{Future directions}

The current studies $(15,16)$ define a central role for Six 1 in the development of diverse tumor types, provide insight into the role of Six1-induced EMT in tumor development, and point toward avenues of research with considerable potential. Six1, as a transcription factor that is little expressed in adult tissues except in tumors, represents an attractive therapeutic target, but selective inhibition of transcription factors can be challenging. However, the new mouse models developed for these studies could be particularly useful for testing and refining appropriately targeted inhibitors. Additionally, defining how Six 1 increases the stem/progenitor cell population, and whether these increases are directly responsible for the subsequent tumor development, could provide insight into the stage of tumor development at which Six1 inhibition might be most effective. Similarly, determination of how Six 1 activates TGF- $\beta$ signaling in breast cells, whether through induction of TGF- $\beta$ itself or through some alternate mechanism, could better define how Six1-induced metastasis might be targeted. Perhaps the most important question will be the extent to which the Six1-induced processes identified here are involved in human breast cancer development and how we can translate these findings for clinical benefit.

\section{Acknowledgments}

D.C. Radisky is supported by the James and Esther King Foundation and by grants from the National Cancer Institute (CA122086 and CA128660) and the Susan G. Komen Breast Cancer Foundation (FAS0703855).

Address correspondence to: Derek C. Radisky, Department of Cancer Biology, Mayo Clinic, 4500 San Pablo Road, Jacksonville, Florida 32224 USA. Phone: (904) 953-6913; Fax: (904) 953-0277; E-mail: radisky.derek@mayo.edu.

1. Acloque, H., Adams, M.S., Fishwick, K., Bronner-Fraser, M., and Nieto, M.A. 2009. Epithelial-mesenchymal transitions: the importance of changing cell state in development and disease. J. Clin. Invest. 119:1438-1449.

2. Hugo, H., et al. 2007. Epithelial--mesenchymal and mesenchymal--epithelial transitions in carcinoma progression. J. Cell Physiol. 213:374-383.

3. Mani, S.A., et al. 2008. The epithelial-mesenchymal transition generates cells with properties of stem cells. Cell. 133:704-715.

4. Morel, A.P., et al. 2008. Generation of breast cancer stem cells through epithelial-mesenchymal transition. PLOS ONE. 3:e2888.

5. Santisteban, M., et al. 2009. Immune-induced epithelial to mesenchymal transition in vivo generates breast cancer stem cells. Cancer Res. 69:2887-2895.

6. Kakarala, M., and Wicha, M.S. 2008. Implications of the cancer stem-cell hypothesis for breast cancer prevention and therapy. J. Clin. Oncol. 
26:2813-2820

7. Barrallo-Gimeno, A., and Nieto, M.A. 2005. The Snail genes as inducers of cell movement and survival: implications in development and cancer. Development. 132:3151-3161.

8. Fuchs, E., Tumbar, T., and Guasch, G. 2004. Socializing with the neighbors: stem cells and their niche. Cell. 116:769-778.

9. Lapidot, T., et al. 1994. A cell initiating human acute myeloid leukaemia after transplantation into SCID mice. Nature. 367:645-648.

10. Al-Haji, M., Wicha, M.S., Benito-Hernandez, A., Morrison, S.J., and Clarke, M.F. 2003. Prospective identification of tumorigenic breast cancer cells. Proc. Natl. Acad. Sci. U. S. A. 100:3983-3988.

11. O'Brien, C.A., Pollett, A., Gallinger, S., and Dick, J.E. 2007. A human colon cancer cell capable of initiating tumour growth in immunodeficient mice.
Nature. 445:106-110.

12. Hermann, P.C., et al. 2007. Distinct populations of cancer stem cells determine tumor growth and metastatic activity in human pancreatic cancer. Cell Stem Cell. 1:313-323.

13. Ricci-Vitiani, L., et al. 2007. Identification and expansion of human colon-cancer-initiating cells. Nature. 445:111-115.

14. Singh, S.K., et al. 2004. Identification of human brain tumour initiating cells. Nature. 432:396-401.

15. McCoy, E.L., et al. 2009. Six1 expands the mouse mammary epithelial stem/progenitor cell pool and induces mammary tumors that undergo epithelial-mesenchymal transition. J. Clin. Invest. 119:2663-2677.

16. Micalizzi, D.S., et al. 2009. The Six1 homeoprotein induces human mammary carcinoma cells to undergo epithelial-mesenchymal transition and metastasis in mice through increasing TGF- $\beta$ signaling. J. Clin. Invest. 119:2678-2690.

17. Abate-Shen, C. 2002. Deregulated homeobox gene expression in cancer: cause or consequence? Nat. Rev. Cancer. 2:777-785.

18. Ford, H.L., Kabingu, E.N., Bump, E.A., Mutter, G.L., and Pardee, A.B. 1998. Abrogation of the G2 cell cycle checkpoint associated with overexpression of HSIX1: a possible mechanism of breast carcinogenesis. Proc. Natl. Acad. Sci. U. S. A. 95:12608-12613.

19. Reichenberger, K.J., Coletta, R.D., Schulte, A.P., Varella-Garcia, M., and Ford, H.L. 2005. Gene amplification is a mechanism of Six 1 overexpression in breast cancer. Cancer Res. 65:2668-2675.

20. Coletta, R.D., et al. 2008. Six1 overexpression in mammary cells induces genomic instability and is sufficient for malignant transformation. Cancer Res. 68:2204-2213.

\section{Endocytic control of ion channel density as a target for cardiovascular disease}

\section{Gail A. Robertson}

Department of Physiology, University of Wisconsin — Madison, Madison, Wisconsin, USA.

\begin{abstract}
Ion channels encoded by the human ether-a-go-go-related gene (HERG) give rise to the rapidly activating delayed rectifier $\mathrm{K}^{+}$current $\left(\mathrm{I}_{\mathrm{Kr}}\right)$, the perturbation of which causes ventricular arrhythmias associated with inherited and acquired long QT syndrome. Electrolyte imbalances, such as reduced serum $\mathrm{K}^{+}$levels (hypokalemia), also trigger these potentially fatal arrhythmias. In this issue of the JCI, Guo et al. report that physiological levels of serum $\mathrm{K}^{+}$ are required to maintain normal HERG surface density in HEK 293 cells and $I_{K r}$ in rabbit cardiomyocytes. They found that hypokalemia evoked HERG channel ubiquitination, enhanced internalization via endocytosis, and ultimately degradation at the lysosome, thus identifying unbridled turnover as a mechanism of hypokalemia-induced arrhythmia. But too little channel turnover can also cause disease, as suggested by Kruse et al. in a study also in this issue. The authors identified mutations in TRPM4 - a nonselective cation channel - in a large family with progressive familial heart block type I and showed that these mutations prevented channel internalization (see the related articles beginning on pages 2745 and 2737, respectively).
\end{abstract}

How can a drop in serum $\mathrm{K}^{+}$levels cause sudden cardiac death? Hypokalemia has long been recognized as a risk factor for potentially catastrophic Torsades de pointes $(\mathrm{TdP})$ ventricular arrhythmias $(1,2)$. TdP is often associated with long QT syndrome, characterized by delayed repolarization after excitation of the heart and a corresponding increase of the QT interval on the ECG (Figure 1). Delayed repolarization arises when ventricular action potentials are prolonged, leading to electrical instabilities at both the cellular and the tissue level that

Conflict of interest: The author has declared that no conflict of interest exists.

Citation for this article: J. Clin. Invest. 119:2531-2534 (2009). doi:10.1172/JCI40427. trigger arrhythmia. A critical determinant of action potential duration (APD) is the so-called rapidly activating delayed rectifier $\mathrm{K}^{+}$channel current $\left(\mathrm{I}_{\mathrm{Kr}}\right.$; ref. 3$)$. Sanguinetti and colleagues demonstrated that $\mathrm{I}_{\mathrm{Kr}}$ block causes APD prolongation in ventricular myocytes (3), and $\mathrm{I}_{\mathrm{Kr}}$ amplitude paradoxically diminishes in low levels of extracellular $\mathrm{K}^{+}$concentration $\left(\left[\mathrm{K}^{+}\right]_{\mathrm{o}}\right)$ despite increased concentration gradient and driving force (4). Thus, $\mathrm{I}_{\mathrm{Kr}}$ emerged as a potential target for hypokalemia-induced TdP.

When it was subsequently determined that $\mathrm{I}_{\mathrm{Kr}}$ was produced by assemblies of subunits encoded by the human ether-a-go-gorelated gene (HERG; refs. 5, 6), analysis of gating mechanisms in the heterologously expressed HERG channel led to the first proposals for a mechanism of hypokalemiainduced reduction in $\mathrm{I}_{\mathrm{Kr}}$. The early studies showed that HERG channels respond to a depolarizing voltage command by entering a stable, inactivated state that suppresses the current; upon repolarization, inactivation recovers to unleash a large resurgent tail current (Figure 2A and refs. 5, 6). During a ventricular action potential, these gating transitions work together to ensure that the resurgent current peaks late and contributes to phase 3 repolarization (Figure $2 \mathrm{~B}$ and ref. 7 ). The suppression at positive voltages occurs by a C-type inactivation mechanism in which the conducting path collapses, a process enhanced in low $\left[\mathrm{K}^{+}\right.$。 $(8,9)$. Such a mechanism could explain a reduction in $\mathrm{I}_{\mathrm{Kr}}$ amplitude in conditions of low $\left[\mathrm{K}^{+}\right]_{\mathrm{o}}$ and was thus proposed to mediate hypokalemia-induced arrhythmia (10).

But the issue is not yet settled. The records of HERG currents in response to voltage step changes (e.g., Figure 2A) at room temperature do not readily predict the effects of hypokalemia on the native channel during an action potential at physiological temperatures. The current profile depends on multiple gating processes with distinct voltage, time, and temperature dependencies (5, $6,11)$. Reducing $\left[\mathrm{K}^{+}\right]_{\mathrm{o}}$ will enhance channel inactivation and thus further suppress current at the peak of the action potential, but the increase in driving force will have the opposite effect on current amplitude as the membrane potential begins to repolarize 